\title{
Optimal dipole-field profiles for emittance reduction in storage rings
}

\author{
Chun-xi Wang* and Yusong Wang \\ Argonne National Laboratory, 9700 South Cass Avenue, Argonne, Illinois 60439, USA \\ Yuemei Peng \\ Institute of High Energy Physics, Yuquan Road 19(B), Beijing 100049, China
}

(Received 28 July 2010; published 21 March 2011)

\begin{abstract}
In recent years nonuniform dipoles with bending-radius variation have been studied for reducing storage ring emittance. According to a new minimum-emittance theory, the effects of an arbitrary dipole can be characterized with two parameters determined by the dipole. To have a better idea of the potentials of nonuniform dipoles, here we numerically explore the possible values of these two parameters and associated bending profiles for optimal emittance reduction. Such optimization results provide a useful reference for lattice designs involving nonuniform bending. Simple bending-radius profiles (a short segment of constant radius with linear ramps on the sides) were found to be close to the optimal. Basic beam and lattice properties such as emittance, energy spread, and phase advances are presented based on the optimal dipole solutions.
\end{abstract}

DOI: 10.1103/PhysRevSTAB.14.034001

PACS numbers: 29.27.Bd, 41.75.-i, 29.20.db, 41.85.-p

\section{INTRODUCTION}

Minimizing beam emittance in storage rings is desired due to ever-increasing demands for higher beam quality for both modern synchrotron light sources and damping rings in high-energy linear colliders. In recent years, there have been efforts to reduce the emittance below the well-known theoretical minimum by using dipoles with bending-radius variation [1-5]. The new theoretical minimum emittance with arbitrary dipoles was established [5] as

$$
\epsilon=\frac{C_{q} \gamma^{2}}{J_{x}} \mathcal{F}^{\mathrm{min}}
$$

where $C_{q}=3.84 \times 10^{-13} \mathrm{~m} ; \gamma$ is the Lorentz factor; and $J_{x}$ is the horizontal damping partition number, which we will not consider here. The lattice-dependent factor $\mathcal{F}$ is a function of bending profile as well as lattice type. Three commonly interested lattice types have been studied: (1) lattices with achromatic arcs, which are useful in providing dispersion-free straight sections for light sources, rf cavities, injection/ejection, and so on; (2) lattices without any constraints except for minimizing the natural betatron emittance, which is the figure of merit for damping rings of linear colliders; and (3) lattices without any constraints but minimizing the effective emittance at the straight sections, which is often the figure of merit for light sources because it takes into account the effects of

\footnotetext{
*wangcx@aps.anl.gov

URL: http://www.aps.anl.gov/ wangcx
}

Published by American Physical Society under the terms of the Creative Commons Attribution 3.0 License. Further distribution of this work must maintain attribution to the author(s) and the published article's title, journal citation, and DOI. dispersion and beam energy spread [6]. We label these three lattice types with AME (achromatic minimum emittance), TME (theoretical minimum emittance), and EME (effective minimum emittance), respectively. The minimal $\mathcal{F}$ for these lattices reads

$$
\mathcal{F}^{\text {min }}=2 \sqrt{|A|} \begin{cases}1 & \text { AME } \\ \sqrt{1-c} & \text { TME } \\ \sqrt{\frac{[1+(q+3) q c / 2][1+[(1+\tau) q+3] q c / 2\}}{1+q c}}, & \text { EME, }\end{cases}
$$

where $|A|$ and $c$ are two parameters solely determined by the dipole profile; $\tau=J_{x} / J_{E}$ is the ratio of horizontal to longitudinal damping partition numbers; and the $q$ parameter is determined by the cubic equation

$$
(1+\tau) q^{3}+2(2+\tau) q^{2}+[3+(2+\tau) / c] q+2 / c=0 .
$$

In this paper, we will use the nominal value $\tau=1 / 2$, while the effect of changing $\tau$ has been addressed in [5]. For conventional uniform dipoles of bending angle $\theta$, $2 \sqrt{|A|} \simeq \theta^{3} / 4 \sqrt{15}$ and $c \simeq 8 / 9$ under the usually good small-angle approximation.

It has been shown that it is possible to reduce $|A|$ and increase $c$, thus reducing the minimum emittance, by optimizing the bending-radius profile of dipole magnets. It also becomes clear that the minimum emittance can approach zero mathematically, except for practical limitations due to magnetic field strength and so on. Thus, a natural question is the potential gains in emittance reduction that nonuniform dipoles may provide. A concise answer is practically important in order for machine designers to decide if it is worthwhile to explore such a potential. The fact that an arbitrary dipole can be characterized by only two values $|A|$ and $c$ (thus a single point in $|A|-c$ parameter space instead of a detailed 
bending-radius profile) provides an effective way to investigate and present a clear picture of the potentials of nonuniform dipoles for emittance reduction. In other words, the answer to the question lies in the distribution of dipoles in the $|A|-c$ parameter space, especially the distribution of optimized dipole-field profiles and corresponding emittances. This paper reports an optimization study of dipole-field profiles using genetic-algorithm (GA)-based optimizers.

Genetic-algorithm optimization methods [7] have long been used in the accelerator field [8] and recently attracted attention following a successful application in multiobjective optimization of a photoinjector design [9] and increased availability of parallel computing resources. Since there are two objective parameters to optimize, we choose to compute the Pareto-optimal solutions in the $|A|-c$ parameter space using a multiobjective GA optimizer based on the genetic algorithm with nondominated sorting (NSGA-II) [10]. For convenience, we adopted a MATHEMATICA ${ }^{\mathrm{TM}}$ implementation of this algorithm [11]. As an independent check and further refinement, a single-objective parallel GA package [12] is also used to optimize emittance directly. Good agreement was found for comparable results. A few analytical results for linear bending profiles are given in the Appendix as illustrations and for comparison.

In the following sections, we will first briefly review the minimum-emittance theory for better understanding of this paper, then describe the methods used for this study, and finally present our results in graphs that give a clear picture of the potential to reduce beam emittance with nonuniform dipoles, as well as the optimal bending profiles and basic properties of the lattices for minimum emittance. We will show that the optimal bending profiles consist of a short segment of constant radius with almost linear ramps on the sides, a pleasantly simple profile resulting from optimization of arbitrary profiles. Note that linear profiles have been studied for specific machines $[1,2]$ and in a more general approach [3]. However, there was no proof it is close to optimal. In fact, a pure linear profile is not optimal (see Appendix) due to the lack of an optimized constant-field segment at peak field. Our study provides a proof by numerically deriving the optimal profile.

\section{BRIEF REVIEW OF THEORY}

Here we briefly outline the basic theory developed in [5]. The natural betatron emittance of Eq. (1) is given by

$$
\mathcal{F}=\frac{\left\langle\mathcal{H} /|\rho|^{3}\right\rangle}{\left\langle 1 / \rho^{2}\right\rangle} \equiv\langle\langle\mathcal{H}\rangle\rangle,
$$

where $\langle\langle\cdots\rangle\rangle$ stands for the bending-radius $(\rho)$ weighted average and the well-known dispersion action $\mathcal{H}$ reads

$$
\begin{aligned}
\mathcal{H} & =\gamma \eta^{2}+2 \alpha \eta \eta^{\prime}+\beta \eta^{\prime 2} \\
& =\operatorname{Tr}\left\{\left(\boldsymbol{\eta}_{0} \boldsymbol{\eta}_{0}^{T}+\boldsymbol{\eta}_{0} \hat{\boldsymbol{\xi}}^{T}+\hat{\boldsymbol{\xi}} \boldsymbol{\eta}_{0}^{T}+\hat{\boldsymbol{\xi}} \hat{\boldsymbol{\xi}}^{T}\right) \sigma_{0}^{+}\right\}
\end{aligned}
$$

Hereafter, $\beta, \alpha$, and $\gamma$ are the usual Courant-Snyder parameters; $\boldsymbol{\eta}_{0}=\left[\eta_{0}, \eta_{0}^{\prime}\right]^{T}$ is the initial dispersion vector; $\hat{\boldsymbol{\xi}}=\left[\hat{\xi}, \hat{\xi}^{\prime}\right]^{T}$ is the dispersion generated in the dipole and projected back to the dipole entrance, which relates to the dispersion vector $\boldsymbol{\eta}$ via the linear transfer matrix $M$ as $\boldsymbol{\eta}(s)=M(s)\left[\boldsymbol{\eta}_{0}+\hat{\boldsymbol{\xi}}(s)\right]$; and $\sigma_{0}^{+}$is the symplectic conjugate of the initial normalized beam matrix $\sigma_{0}$. More explicitly,

$$
\begin{gathered}
\sigma^{+} \equiv-J \sigma^{T} J=\left[\begin{array}{cc}
\gamma & \alpha \\
\alpha & \beta
\end{array}\right], \quad \sigma=\left[\begin{array}{cc}
\beta & -\alpha \\
-\alpha & \gamma
\end{array}\right], \\
J=\left[\begin{array}{cc}
0 & 1 \\
-1 & 0
\end{array}\right] .
\end{gathered}
$$

Equations (4) and (5) yield

$$
\mathcal{F}=\operatorname{Tr}\left(G_{0} \sigma_{0}^{+}\right),
$$

where

$$
G_{0}=\check{\rho} \boldsymbol{\eta}_{0} \boldsymbol{\eta}_{0}^{T}+\boldsymbol{\eta}_{0}\langle\langle\hat{\boldsymbol{\xi}}\rangle\rangle^{T}+\langle\langle\hat{\boldsymbol{\xi}}\rangle\rangle \boldsymbol{\eta}_{0}^{T}+\left\langle\left\langle\hat{\xi} \hat{\boldsymbol{\xi}}^{T}\right\rangle\right\rangle,
$$

and $\check{\rho} \equiv\langle\langle 1\rangle\rangle=\left\langle 1 /|\rho|^{3}\right\rangle /\left\langle 1 / \rho^{2}\right\rangle=I_{2}$, a well-known radiation integral.

For a given dipole and initial dispersion, $G_{0}$ is determined and $\mathcal{F}$ is minimized to $2 \sqrt{\left|G_{0}\right|}$ with the optimal lattice parameters given by $\sigma_{0}=G_{0} / \sqrt{\left|G_{0}\right|}$ at the dipole entrance. From Eq. (8) it is easy to see that $\left|G_{0}\right|$ (thus $\mathcal{F}$ ) can be further minimized by choosing the initial dispersion vector along the average of the projected dispersion $\langle\langle\hat{\boldsymbol{\xi}}\rangle\rangle$. Let $\boldsymbol{\eta}_{0}=q\langle\langle\hat{\boldsymbol{\xi}}\rangle\rangle / \check{\rho}$, then $G_{0}$ reduces to

$$
G_{0}=A+\left(q^{2}+2 q\right) B,
$$

where the matrices

$$
A=\left\langle\left\langle\hat{\boldsymbol{\xi}} \hat{\boldsymbol{\xi}}^{T}\right\rangle\right\rangle \quad \text { and } \quad B=\langle\langle\hat{\boldsymbol{\xi}}\rangle\rangle\langle\langle\hat{\boldsymbol{\xi}}\rangle\rangle^{T} / \check{\rho} .
$$

The determinant of Eq. (9) can be reduced to

$$
\begin{aligned}
& \left|G_{0}\right|=|A|\left[1+\left(q^{2}+2 q\right) c\right], \\
& \text { where } c=-\frac{\operatorname{Tr}(J A J B)}{|A|} .
\end{aligned}
$$

Therefore, $\mathcal{F}^{\mathrm{min}}$ is given by $q=0$ for AME and $q=-1$ for the TME lattice, as shown in Eq. (2).

It is clear that the $|A|$ and $c$ parameters are characteristics of a dipole magnet. $\sqrt{|A|}$ is proportional to the magnitude of emittance due to quantum excitation in the dipole, and $c$ reflects the degree of correlation among the excitations, while correlated excitations can be suppressed with proper choice of the initial dispersion.

For light sources the figure of merit is the effective emittance $\epsilon_{\text {eff }}$ at the straight sections that takes energy deviation $\delta$ into account. In terms of phase-space average,

$$
\begin{aligned}
\epsilon_{\mathrm{eff}} & \equiv \sqrt{\left\langle(x+\eta \delta)^{2}\right\rangle\left\langle\left(x^{\prime}+\eta^{\prime} \delta\right)^{2}\right\rangle-\left\langle(x+\eta \delta)\left(x^{\prime}+\eta^{\prime} \delta\right)\right\rangle^{2}} \\
& =\epsilon_{x} \sqrt{1+\mathcal{H}_{\mathrm{ID}} \sigma_{\delta}^{2} / \epsilon_{x}},
\end{aligned}
$$


where $x$ and $x^{\prime}$ are the position and angle of the transverse betatron motions; $\epsilon_{x}$ is the natural betatron emittance. Minimization of this effective emittance is more involved, which yields the $\mathcal{F}^{\text {min }}$ for EME lattice in Eq. (2) and the optimal $q$ given by Eq. (3). Note that this effective emittance assumes no significant collective effects on beamenergy spread.

This theory solved the minimum emittance for a given dipole bending profile. The optimal bending profile is yet to be determined from an infinite number of possibility, which is carried out by this paper.

\section{METHODS}

Before getting into optimization techniques, we address the rationale of our optimization strategy. Although minimizing emittance is the goal, we choose not to minimize $\mathcal{F}$ directly (we did this as an alternative confirmation) because it will depend on lattices in addition to bending profiles. For example, the EME emittance has nontrivial dependence on lattice damping partition. Direct optimization at one damping partition may be of limited use for different partition settings. On the other hand, knowing the possible $|A|$ and $c$ values, it is easy to compute the minimum emittance for any lattices, and thus to optimize emittance over various lattices as well as parameters such as the damping partition numbers [5]. Therefore we choose to simultaneously optimize $|A|$ and $c$, which is solely determined by bending profiles, while smaller $|A|$ and larger $c$ yield smaller emittance. Another reason we choose to optimize $|A|$ and $c$ is that they better reveal the dependence of emittance on the dipole profiles and provide valuable information on potential trade-offs. Based on these, we consider the distribution of optimal $|A|$ and $c$ a more basic and desirable solution of this dipole optimization problem. However, this requires a multiobjective optimization to simultaneously optimize $|A|$ and $c$.

To numerically evaluate the efficacy of nonuniform dipoles for emittance reduction as well as lattice feasibility, we use a uniform dipole $1 \mathrm{~m}$ long with 10-meter bending radius as the reference, and compare it with nonuniform dipoles having the same length and bending angle. The resulting emittance reduction factor and the ratio of initial lattice functions should apply to other dipole parameters, as long as the small-angle approximation is valid, thanks to the scale-invariant property of the theory. This helps to reduce the complexity of the problem dramatically and makes our optimization results valid for general reference (because they depend on only the shape of the bending profile instead of dipole length, field strength, and bending angle).

To evaluate a bending profile, we use a large number of equal-length dipole slices to approximate an arbitrary dipole and use the bending curvature $h(s)=1 / \rho(s)$ to represent a dipole-field profile, where $\rho(s)$ is the bending radius. In this study we explore dipoles without field polarity inversions, considering that field inversions might be too complicated to build. Our computation starts from the basic quantity in the minimum-emittance theory, i.e., the projected dispersion vector $\hat{\xi}=\left[\hat{\xi}, \hat{\xi}^{\prime}\right]^{T}$. To numerically solve for the projected dispersion $\hat{\xi}$ and $\hat{\xi}_{p} \equiv \hat{\xi}^{\prime}$, we directly solve the first-order differential equations $\hat{\boldsymbol{\xi}}^{\prime}=$ $M^{-1}[0, h]^{T}$ using the transfer matrix $M$ and initial condition $\hat{\boldsymbol{\xi}}_{0}=0$, i.e.,

$$
\hat{\xi}^{\prime}=-h M_{12}, \quad \hat{\xi}_{p}^{\prime}=h M_{11}, \quad \hat{\xi}(0)=\hat{\xi}_{p}(0)=0 .
$$

From $\hat{\boldsymbol{\xi}}$ we can compute the matrices $A, B$, and the parameter $c$ as defined in Eqs. (10) and (11), where averaging over the dipole is done by numerical integration.

The optimization is carried out with GA optimizers. A population of 100 or so individual bending profiles are initialized randomly with each individual having a chromosome length equal to the number of dipole slices. The population is then evolved using the elitist multiobjective optimizer based on the genetic algorithm with nondominated sorting (NSGA-II). After sufficient generations, the Pareto-optimal front is obtained. The emittance and optimal lattice parameters are computed for each individual on the optimal front, and results are summarized in graphs. As an independent check and for better converging efficiency, a single-objective parallel GA package (PGApack) is used to optimize the AME, TME, and EME emittances directly, which should reproduce the corresponding extreme points of emittance curves obtained from the optimal-front population.

To look into the lattice feasibility of an optimized result, we compute some basic lattice properties such as betatron phase advances, the ratio of initial lattice parameters of the optimized profile to the reference uniform dipole. Such information should hint at the difficulty of realizing an optimal solution, although designing a feasible lattice is much more involved. The lattice-parameter distribution on the Pareto-optimal front provides a useful way to make a trade-off between emittance reduction and lattice difficulty. Our goal is to obtain a clear picture of the landscape for emittance reduction using nonuniform dipoles.

In addition to numerical optimization, we analytically worked out expressions for a few simple profiles studied in the literature. Although not essential for this paper, such exercises help to illuminate the framework and provide connections to existing works. Performance of these profiles is presented for comparison. This nonessential material is given in the Appendix.

\section{OPTIMIZATION RESULTS}

\section{A. Pareto-optimal solutions}

Pareto-optimal solutions in the objective space consist of solutions that are optimum in any objective such that it is impossible to improve one objective without worsening others. Such a set of solutions is often referred to as a 


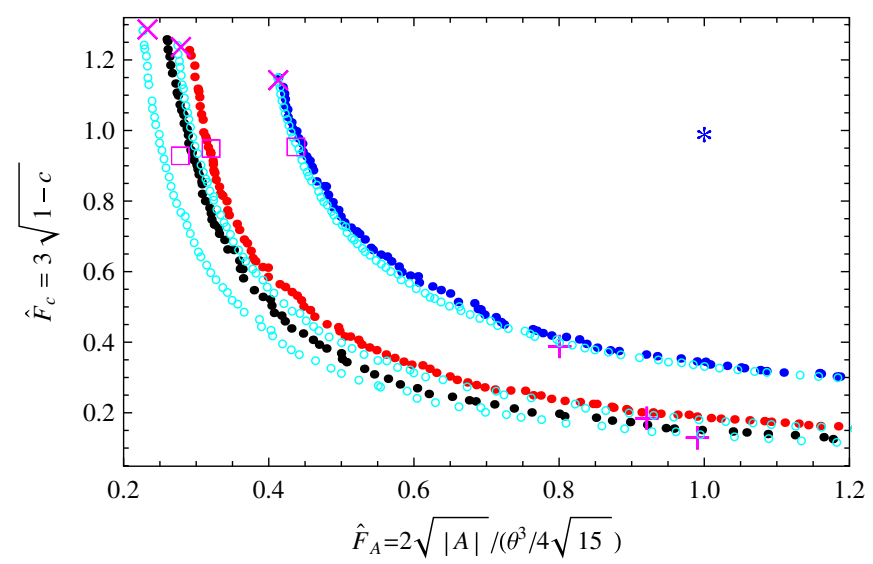

FIG. 1. Pareto-optimal solutions in the objective space. The colored dots are multiobjective optimizations of 33-slice dipoles whose maximum field is higher than the reference dipole by a factor of 2 (blue), 4 (red), and 6 (black). The markers (pink) represent results of single-objective PGA optimization of AME ( $\times, 65$ slices), TME (+ , 65 slices), and EME ( $\square, 33$ slices). The blue star marks the uniform dipole. The cyan circles are optimizations based on simplified field profiles (linear-ramp model discussed later). Longer tails continuing off the lower-right corner are not shown.

Pareto front, which reveals compromises among multiple objectives to be optimized. In our case, we need to minimize $|A|$ and maximize $c$ for minimal emittance. In fact, we choose to minimize both $2 \sqrt{|A|}$ and $\sqrt{1-c}$, normalized by the values of the reference uniform dipole, i.e., minimize $\hat{\mathcal{F}}_{A}=2 \sqrt{|A|} /\left(\theta^{3} / 4 \sqrt{15}\right)$ and $\hat{\mathcal{F}}_{c}=3 \sqrt{1-c}$. The resulting Pareto-optimal solutions under several maximum field strengths are plotted in Fig. 1. It contains multiobjective optimizations based on 33-slice dipoles (colored dots) and a simplified linear-ramp model (cyan circles), as well as single-objective optimizations of 33-slice (pink squares) and 65 -slice (pink + and $\times$ ) dipoles. Each point in Fig. 1 represents a specific profile. We considered three peak-field values, which yield three families of Pareto-optimal profiles represented by the blue, red, and black colors. Three optimization methods are used to ensure convergence to the optimal solutions. At low peak field, all three optimizations (blue dots, cyan circles, and pink markers) converged. At higher peak field, 33-slice optimizations (red dots and pick square) converged but fall behind the optimal, while the 65 -slice optimization yielded the optimal solutions. At even higher peak field, the multiobjective optimization (black dots) falls behind the single-objective optimization (pink square) using the same 33 slices, and further behind the optimal solution. Multiobjective optimization of the linearramp model (see Sec. V) converged to the optimal solutions because the model is very close to the optimal, does not suffer from multislice approximation, and involves only a few parameters.

From the optimal solutions in Fig. 1 and the emittance formula, it is easy to see the potential emittance reduction



FIG. 2. Emittance reduction factors for AME, TME, and EME lattices based on the optimal solutions (cyan circles) in Fig. 1. Again, the maximum field strength is a factor of 2 (blue), 4 (red), and 6 (black). The pink markers on the curves indicate PGA results and the lattice types of the associated curves as AME $(\times)$, TME (+), and EME ( $\square$ ). The differences in EME at high peak fields are due to using 33 instead of 65 or more slices. Note that there are three emittance reduction factors for each dipole profile (a cyan circle in Fig. 1) corresponding to the AME, TME, and EME lattice conditions, respectively.

using nonuniform dipoles. To be more explicit, we computed the emittance reduction factor for AME, TME, and EME lattices using the optimal solutions and summarized the results in Fig. 2. In this and several other plots, we use the "normalized $c$-factor" $\hat{\mathcal{F}}_{c}$ as a tag to differentiate the solutions in Fig. 1. The behaviors of AME and EME are similar, and both are dominated by the $2 \sqrt{|A|}$ factor. This is good since both AME and EME are of interest to light sources, and the similarity may allow some flexibility in switching lattices. On the other hand, the TME reduction is much larger and dominated by the $\sqrt{1-c}$ factor. Note that dipoles optimized for TME are not effective at all for AME and EME. This plot suggests that nonuniform dipoles will be more effective for damping rings since they favor TME lattices [13]. However, such considerations are based on double-bend lattices. For multibend lattices, the inner bends can take advantage of the large emittance reduction factor of symmetric TME bends, which makes nonuniform dipoles more attractive to light sources.

\section{B. Optimal field profiles}

The optimal bending curvatures (field profiles) for some special cases are shown in Figs. 3 and 4. Figure 3 plots the optimal profiles for minimal TME, corresponding to the solutions marked by the pink "+" in Fig. 1. It shows increasingly stronger and shorter constant field at the center (providing most of the bending) and symmetric tails with much weaker fields to preserve the total bending angle. From the profile symmetry we can gauge the quality 


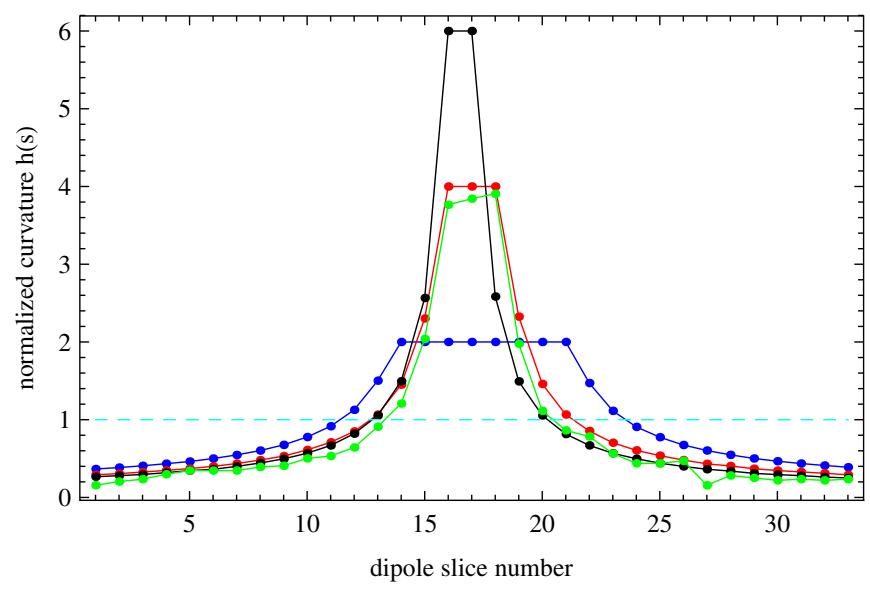

FIG. 3. Optimal bending field profiles (from PGA optimizations) for minimal TME at maximum field strength 2 (blue), 4 (red), and 6 (black) times higher than the reference dipole (dash). The green curve is a multiobjective optimization, which is not fully optimized, with a few percent lower performance. The cyan dashed line is the reference uniform dipole. Note that, in this and the next two graphs, the curves do not represent the field but simply provide a guidance for the actual piecewise constant field represented by the dots.

of the optimization. Asymmetry due to the finite slice number is apparent in the blue and black curves, whose effect on the emittance is small. For comparison, a multiobjective optimization result is indicated by the green curve. Note that emittance reduction is not very sensitive to field errors. Figure 4 plots the optimal profiles for minimal AME, TME, and EME at 4 times higher maximum field. It clearly shows that the optimal profiles are different for different types of lattices, although the AME and EME are similar, with the constant-field segment close to the front of the dipole. Note that the optimal dipole for EME is effectively shorter by about $12 \%$.

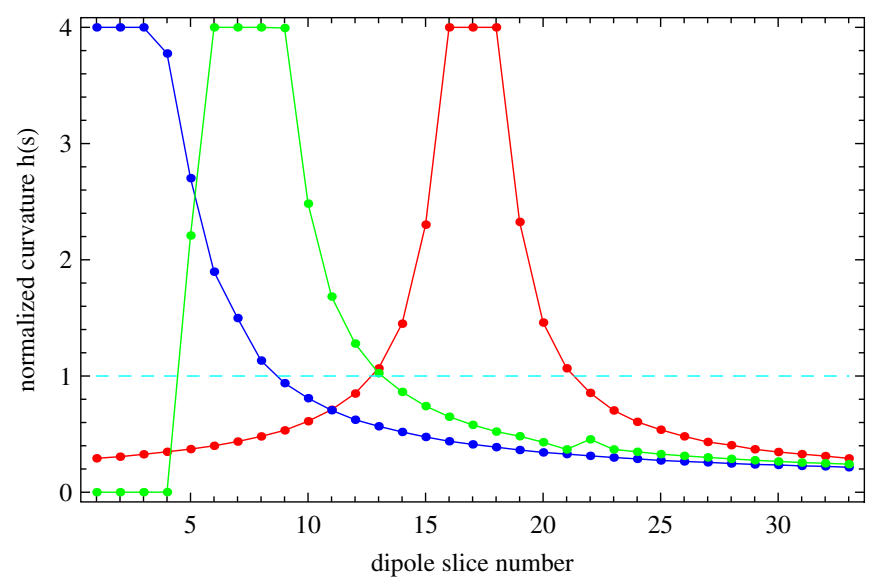

FIG. 4. Optimal bending field profiles for minimal AME (blue), TME (red), and EME (green) at maximum field strength 4 times higher than the reference dipole (dash).

\section{LINEAR-RAMP MODEL OF OPTIMAL PROFILES}

Examination of the optimal bending-radius profiles reveals that the optimal profiles consist of a shorter and stronger constant-field segment with mostly linear ramps in bending radius, as shown in Fig. 5. The length and position of the constant-field segment vary for different types of lattices. Such a picture simplifies the optimal bending profiles and allows a simple model with at most four parameters. A small number of parameters can be important when optimizing nonuniform dipoles together with many other parameters in a lattice design. It also reduces the uncertainty/noises in optimizations. Furthermore, the simplified model does not suffer from multislice approximation (e.g., the lower performance at 4-times and 6-times peak field in Figs. 1 and 2; the asymmetry in Fig. 3). This simple linear-ramp model also opens up the possibility for analytical solutions, which are not pursued here.

To test this linear-ramp model, we rerun the optimization with it and plot the results (cyan circles) in Fig. 1 to compare with the results of general multislice optimizations. We see that the linear-ramp model performs just as well at 2-times peak field, better than the 33-slice optimizations at higher peak fields, and consistent with 65 -slice PGA optimizations. Optimization using this simplified model is much more efficient due to the small number of control parameters. For the results in Fig. 1, we used 50000 generations for the multislice model versus 5000 generations for the linear-ramp model, using similar optimization parameters such as the mutation and crossover rates. (We made no effort to improve the optimization efficiency in either case.) Because of its performance, most of the multiobjective optimization results presented in this paper (except for those in Figs. 1 and 3) are based on the linear-ramp model.

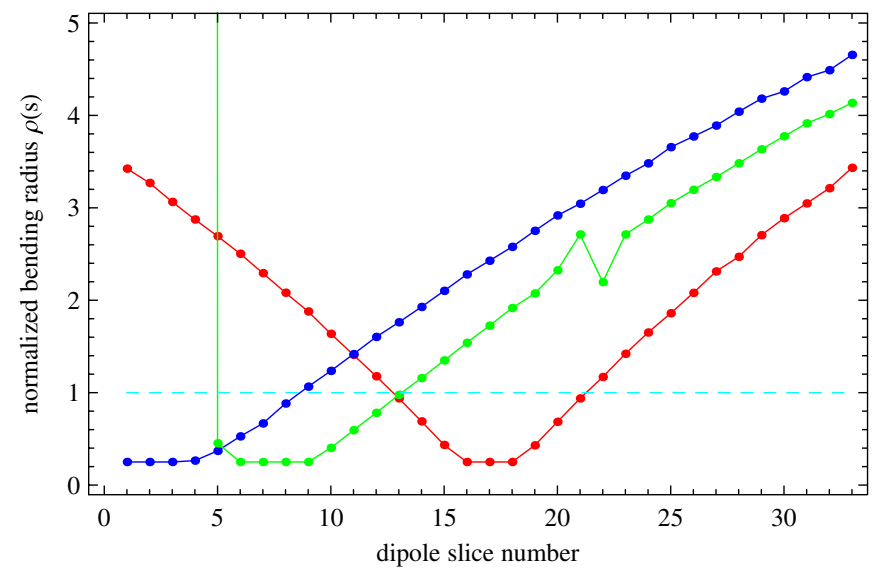

FIG. 5. Optimal bending-radius profiles corresponding to the curvature profiles in Fig. 4 for AME (blue), TME (red), and EME (green) lattices. The ramps are fairly close to linear. The glitch in the green curve is a minor imperfection in optimization. 


\section{LATTICE PROPERTIES FOR OPTIMAL EMITTANCE}

In this section, we present some basic lattice properties associated with the optimal dipole solutions, such as beam energy spread, horizontal betatron phase advances, beta function, and dispersion. Such information may give some idea of the difficulty in implementing lattices of the optimal solutions and can be useful when considering trade-offs between emittance and lattice properties. In this section, we have used the optimized linear-ramp profiles, which tend to have less noise due to the much smaller parameter space.

\section{A. Dispersion action $\mathcal{H}$ inside dipoles}

To better appreciate the emittance reduction mechanism in nonuniform dipoles, Fig. 6 plots the dispersion action $\mathcal{H}$ inside the dipoles shown in Figs. 4 and 5 for the optimal AME, TME, and EME at 4-times peak field. For comparison, the dispersion actions in a uniform dipole are shown as dashed lines. Clearly, the dispersion action is reduced in high-field regions by tailing the field profiles and corresponding dispersion actions, thus reducing the weighted average $\left\langle\mathcal{H}|h|^{3}\right\rangle$, which is the radiation integral $I_{5}$. Furthermore, nonuniform bending increases the radiation integral $I_{2}=\left\langle h^{2}\right\rangle$ by a factor 2.47 (AME), 2.16 (TME), and 2.56 (EME). Thus, the emittance (proportional to $I_{5} / I_{2}$ ) is reduced. The gain from the $I_{5}$ reduction is significant for TME, but not so much for AME and EME.

Because of the increase in $I_{2}$, radiation loss will increase proportionally, which will result in higher demand on $\mathrm{rf}$ power and stronger radiation damping.

\section{B. Energy spread}

Beside emittance reduction, bending field variation will increase the radiation integral $I_{3}=\left\langle|h|^{3}\right\rangle$ more than $I_{2}$,

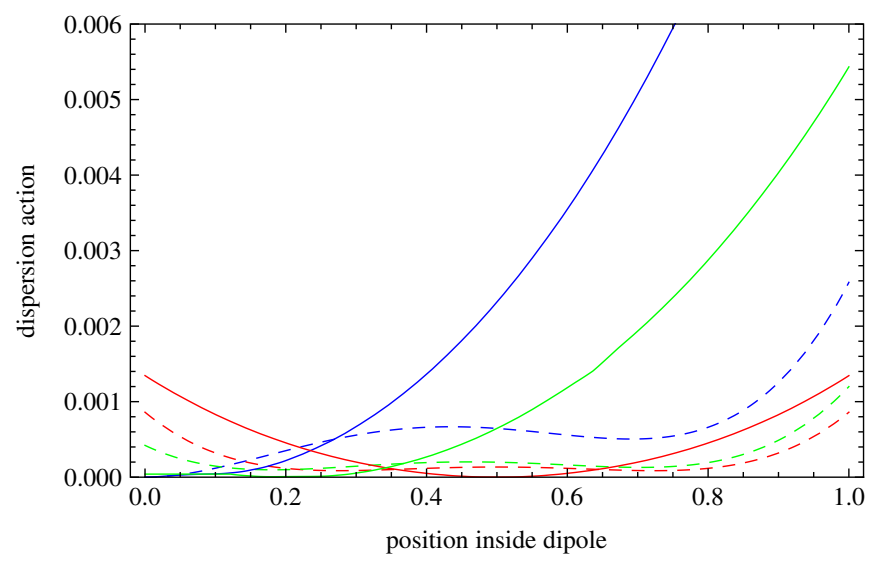

FIG. 6. Dispersion action inside the optimal dipoles shown in Figs. 4 and 5 for AME (blue), TME (red), and EME (green). Dashed curves are corresponding dispersion actions in the reference uniform dipole.

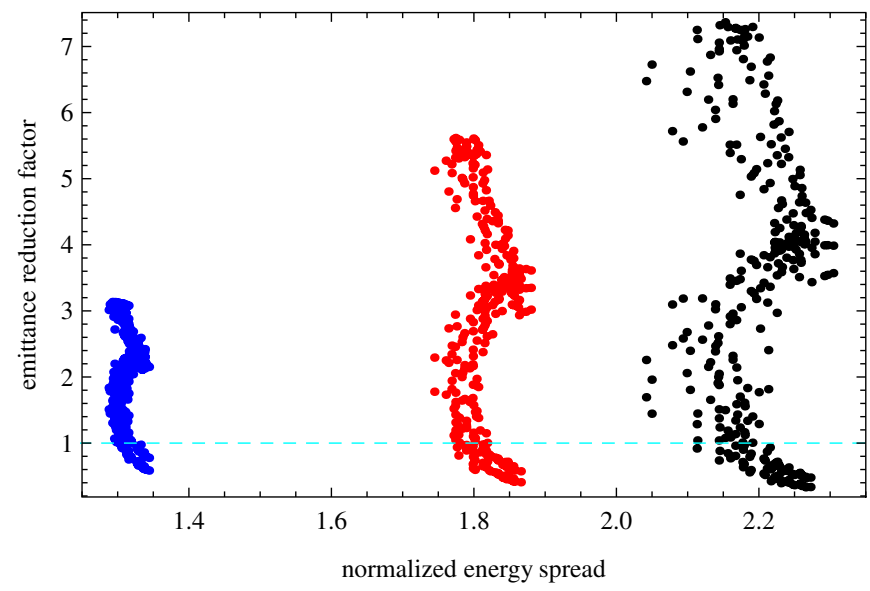

FIG. 7. Relative increases in beam energy spread for the optimal solutions in Fig. 1, using the same color scheme for the maximum field strength 2 (blue), 4 (red), and 6 (black). For each solution, three dots are plotted corresponding to the emittance reduction factors for AME, TME, and EME. (We choose not to use different markers for different lattices to avoid overlapping. However, from Fig. 2, it is clear that the upper branch is from TME lattice.)

thus increasing beam energy spread proportional to $\sqrt{I_{3} / I_{2}}$. The effect of energy spread on synchrotron light sources has been taken into account by minimizing the effective emittance. (Note that the effective emittance assumes no energy-spread increase from collective effects.) However, large energy spread may worsen chromatic effects and challenge momentum aperture. To see the relative increase in beam energy spread, Fig. 7 plots the AME, TME, and EME emittance reduction factor versus the energy spread for the Pareto-optimal solutions in Fig. 1. The increase in energy spread might limit the usefulness of nonuniform dipoles in some machines.

\section{Phase advances in optimal lattices}

To obtain the minimum emittance of an optimal dipole profile as shown in Fig. 2, lattice parameters such as beta function, phase advance, and dispersion must match the optimal values. The phase advances in the inner dispersion matching sections and the straight section are determined by the dispersion matching (except for a dispersion-free straight section). These phase advances will indicate required focusing. Larger phase advances result in stronger focusing and associated increase in natural chromaticity.

For each Pareto-optimal solution in Fig. 1, we computed the phase advance in the dipole under AME, TME, and EME lattice conditions; the results are shown in Fig. 8. On the right-hand side, where AME- and EME-favored dipole profiles are located, we see negligible increases over the $150^{\circ}$ of a uniform dipole. TME-favored profiles result in larger phase advances, which fall off sharply on the left edges (similar to the TME emittance curve in Fig. 2). 


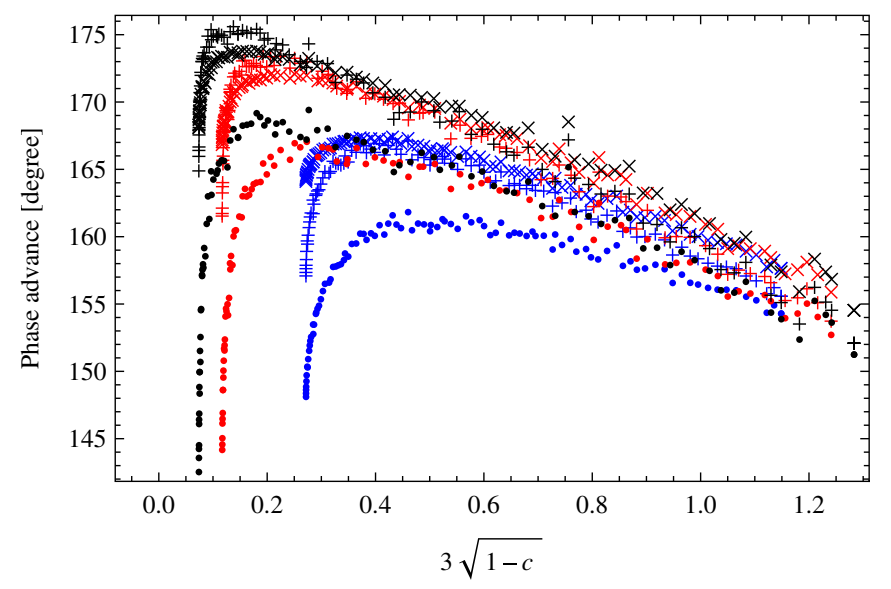

FIG. 8. Phase advances within an optimal dipole for AME $(\times)$, $\operatorname{TME}(+)$, and $\operatorname{EME}(\bullet)$ under 2 (blue), 4 (red), and 6 (black) times higher peak field. The corresponding phase advances for the reference uniform dipole are $157^{\circ}, 151^{\circ}$, and $150^{\circ}$, respectively.

Figure 9 plots the minimum phase advances in the inner section of a symmetric double-bend cell based on the Pareto-profiles under AME, TME, and EME conditions. There are significant increases in phase advances from the conventional uniform double-bend cell except for the left edge (where the TME emittance reduction is compromised but still appreciable).

Figure 10 plots the minimum phase advances in the straight section (for insertion devices), assuming the same dipole at both ends. Note that for the AME lattice, there is no constraint on the phase advance from dispersion matching. For EME lattices, there is little change in this phase advance compared with the uniform dipole lattice. However, there are large increases for TME lattices.

So far we have considered phase advances inside and between the same dipoles in symmetric double-bend cells.

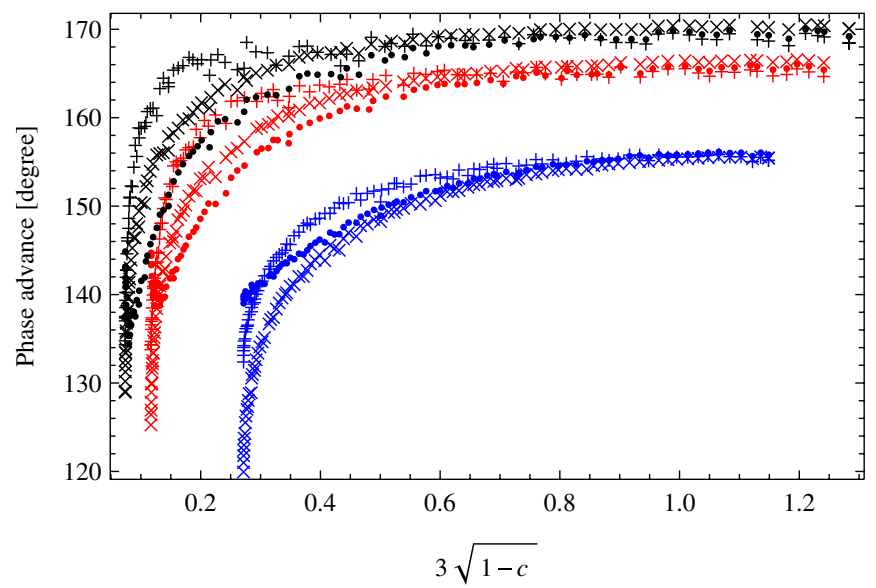

FIG. 9. Phase advances in the dispersion matching section in symmetric double-bend cells for AME (X), TME (+), and EME $(\bullet)$ under three different peak fields 2 (blue), 4 (red), and 6 (black). Phase advances for uniform dipoles are $122^{\circ}, 133^{\circ}$, and $133^{\circ}$, respectively.

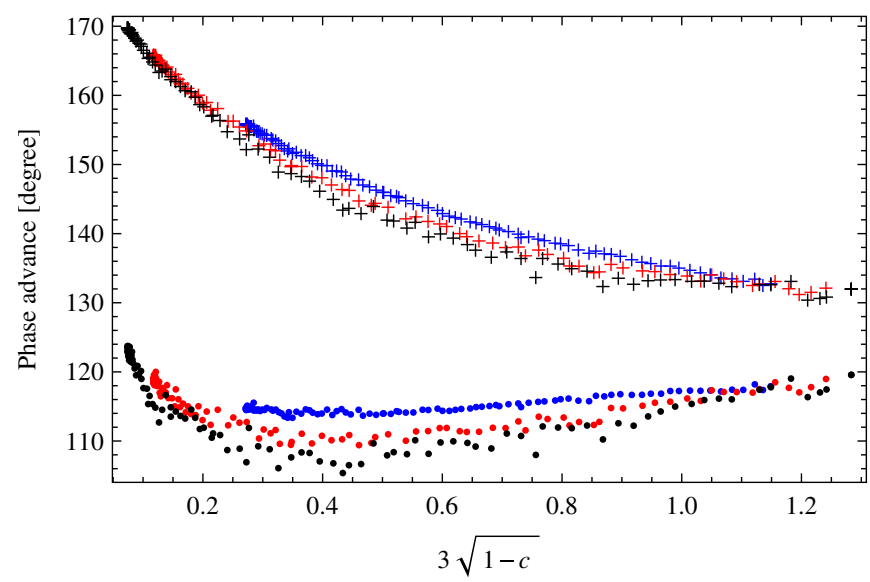

FIG. 10. Phase advances in the straight section for TME $(+)$, and $\operatorname{EME}(\bullet)$ under different peak fields 2 (blue), 4 (red), and 6 (black). Phase advances for uniform dipoles are $133^{\circ}$ and $115^{\circ}$, respectively.

For multibend cells, one may want to match AME or EME dipoles at the cell ends to TME dipoles inside. For this purpose, Fig. 11 plots the minimum phase advances required to match a dipole from the Pareto solutions to the ideal TME dipole of the same peak field. The results are similar to the phase advances of double-bend dispersion matching sections in Fig. 9.

One complication in dispersion matching for multibend lattices is to maintain the same dispersion action $\mathcal{H}$ at the dipole ends. Otherwise, it is impossible to match the dispersion [14], since dispersion action is conserved in any dipole-free sections. To examine this mismatch for the Pareto solutions, Fig. 12 plots the ratio of dispersion actions at the exit of the optimal dipole solutions in Fig. 1 to the dispersion action at the entrance of the ideal TME dipole as shown in Fig. 3. The large values on the

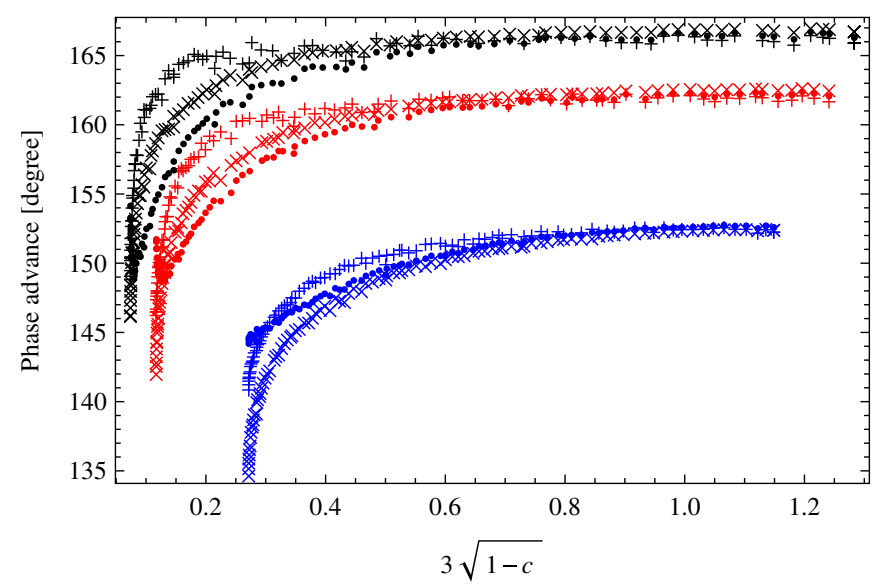

FIG. 11. Phase advances needed for dispersion matching from $\operatorname{AME}(\times)$, TME $(+)$, and $\operatorname{EME~}(\bullet)$ dipoles to the TME dipole under different peak fields 2 (blue), 4 (red), and 6 (black). Phase advances for uniform dipoles are $128^{\circ}, 133^{\circ}$, and $133^{\circ}$, respectively. 


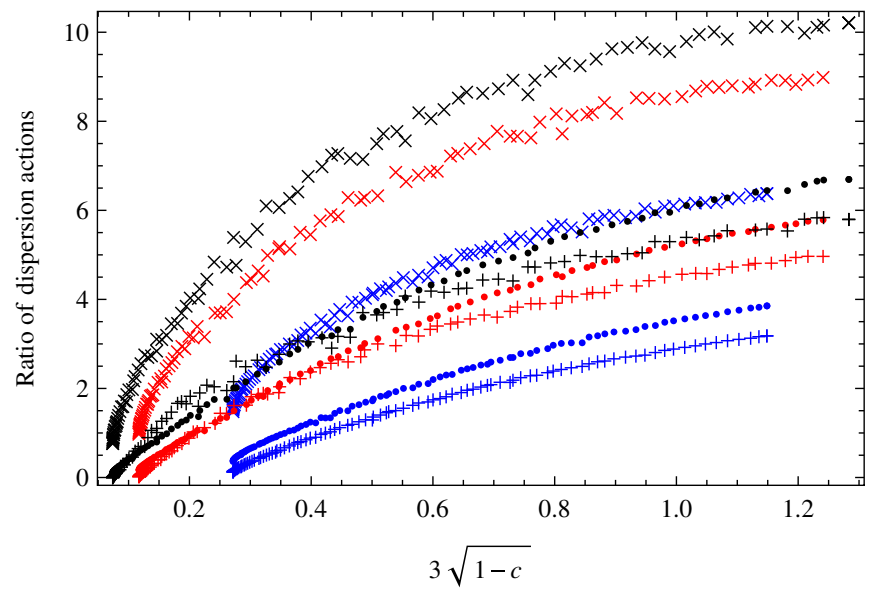

FIG. 12. Ratio of dispersion action of $\operatorname{AME}(\times)$, TME $(+)$, and EME $(\bullet)$ dipoles to the TME dipole at different peak-field factor of 2 (blue), 4 (red), and 6 (black). The corresponding ratios for uniform dipoles are 3,1 , and 1.4 , respectively.

right-hand side reflect the efficacy of TME-optimized dipoles in reducing dispersion action. This indicates that the central TME dipoles need to be much stronger/longer than the end dipoles, which can be beneficial to multibend lightsource lattices.

\section{Initial beta function}

Figure 13 plots the emittances versus the relative change in initial beta function at the dipole entrance. It shows that TME reduction requires larger initial beta, while AME and EME favor smaller initial beta. It is encouraging to see that significant emittance reduction (not far from the optimum) can be achieved without large changes in the initial beta function. Furthermore, Fig. 14 plots the relative change in initial beta functions versus the initial alpha functions. It shows that, for solutions close to the optimal for TME, the

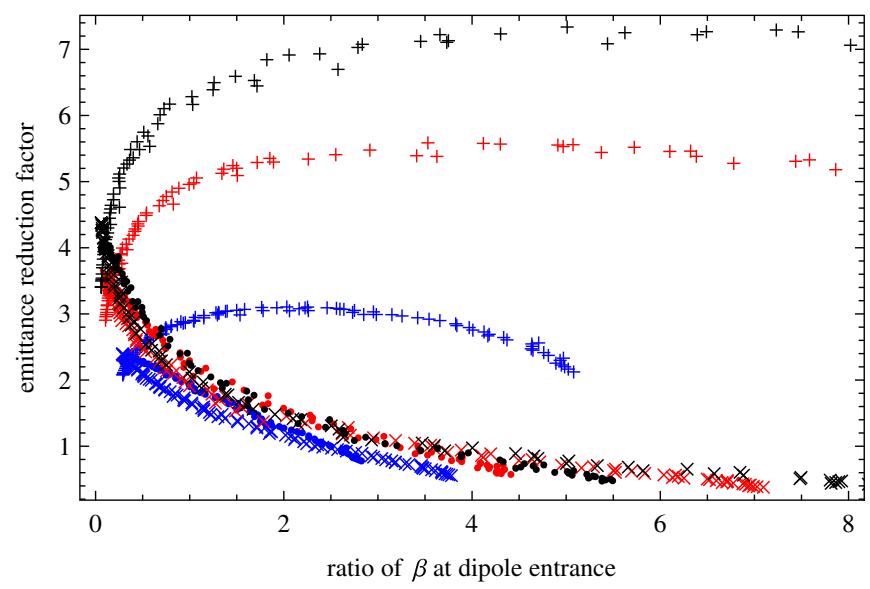

FIG. 13. Relative changes in initial $\beta$ functions at the dipole entrance for the optimal solutions. Lattices are marked by AME $(\times)$, TME $(+)$, and EME $(\bullet)$. Peak-field strength factors are 2 (blue), 4 (red), and 6 (black).

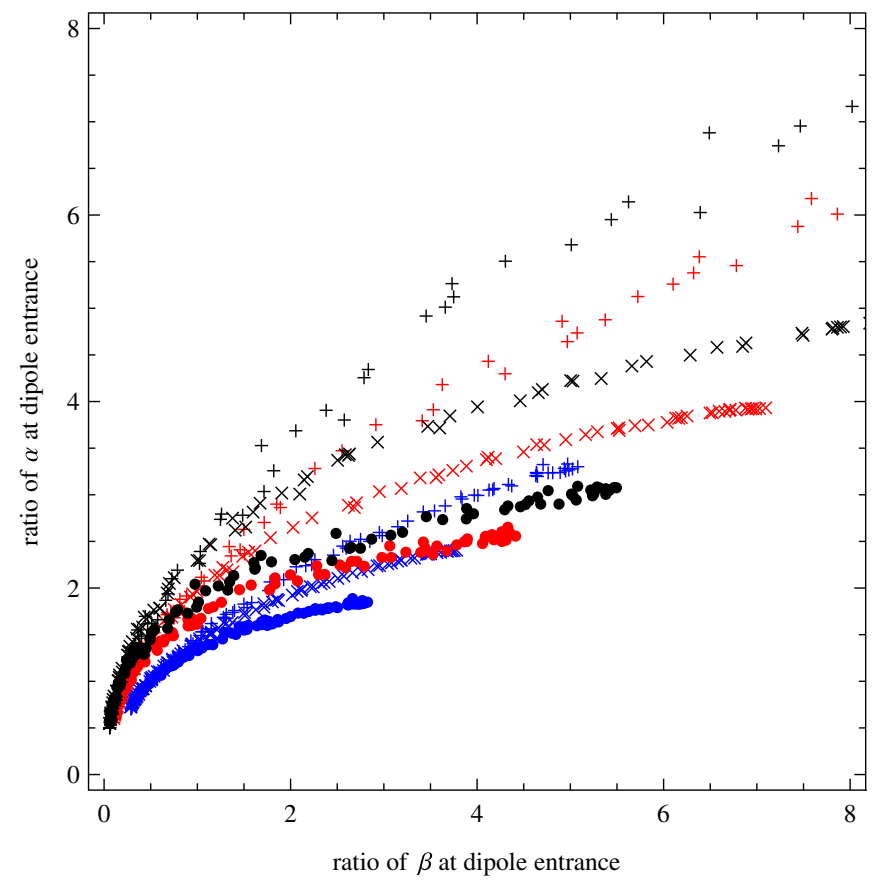

FIG. 14. Relative changes in initial $\beta$ and $\alpha$ functions at the dipole entrance for the optimal solutions. Lattices are marked by AME $(\times)$, TME $(+)$, and EME $(\bullet)$. Peak-field strength factors are 2 (blue), 4 (red), and 6 (black).

changes in beta and alpha functions are more or less proportional, which indicates that the TME emittance reduction may be tolerable to deviations in initial Twiss parameters (see Fig. 1 in [5]). On the other hand, for AME and EME, the reduction in initial beta tends to be much larger than the variation in initial alpha, which might be harder to accommodate. Note that, even with uniform dipoles, beta and alpha functions are often different from the optimum values in order to lower chromaticity. In both Figs. 13 and 14, there are solutions with larger beta and alpha continuing off the plot, which correspond to the falling edges on the left in Fig. 2. They are excluded to make the plots less clustered at the low end.

\section{E. Dispersion and dispersion action}

Since effective emittance has already taken into account dispersion effects on light sources, our concern for dispersion here is mainly for chromaticity correction, because dispersion has direct impact on the required sextupole strength for chromaticity correction and associated nonlinearity. Although the dispersions at the dipole ends are determined for the optimal solutions, the dispersions at sextupoles can be very different and depend on more details in the lattice design. Thus, we will not show the dispersions here; instead we look for something more relevant.

For simplicity and clarity, we limit our discussion to symmetric double-bend cells, in which the dispersion reaches its maximum at the center where chromatic 


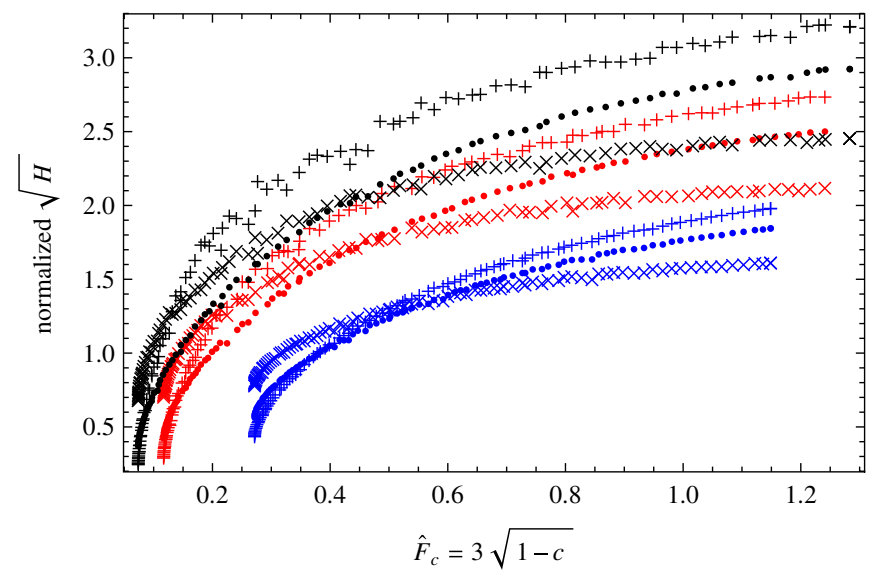

FIG. 15. Relative changes in the square root of dispersion action in the inner matching section of symmetric double-bend cells for the optimal solutions. Lattices are marked by $\mathrm{AME}(\times)$, TME $(+)$, and EME $(\bullet)$. Peak-field strength factors are 2 (blue), 4 (red), and 6 (black).

sextupoles are usually located. To the first order, a sextupole contribution to chromaticity is proportional to both dispersion and beta function, thus we examine $\beta \eta$ at the double-bend center. At this symmetric point, $\eta^{\prime}=\alpha=0$ and the dispersion action reduces to $\mathcal{H}=(\eta / \sqrt{\beta})^{2}$; thus $\beta \eta=\sqrt{\beta^{3} \mathcal{H}}$. Since the dispersion action is conserved, it can be computed at the dipole end facing the sextupoles. Without further detail on lattice design, the beta function cannot be determined. Nonetheless, it is still informative to examine the change in the dispersion action relative to the uniform dipole. Figure 15 plots the normalized $\sqrt{\mathcal{H}}$. It gives the relative change in sextupole strength (weaker for larger $\sqrt{\mathcal{H}}$ ), assuming the same beta function. It shows beneficial gains for AME and EME lattices (on the right) and small loss for the TME lattice (on the left). This indicates that the effects on sextupole strength and chromatic correction are probably manageable.

\section{CONCLUDING REMARKS}

Using genetic-algorithm-based multiobjective as well as single-objective optimizations of arbitrary bending profiles without field reversion, we established the optimal profiles for emittance minimization and the corresponding theoretical minimum emittances for AME, TME, and EME lattices. We showed that the optimal bending-radius profiles consist of a short constant segment at the smallest bending radius and almost linear ramps on both sides. Compared to uniform dipoles, optimal nonuniform dipoles can yield significantly smaller theoretical minimum emittance, especially for TME lattices. We further examined the increase in energy spread due to bending-radius variation and other basic lattice properties. Despite an energyspread increase, effective emittance for light sources can still be reduced. Nonetheless, larger energy spread might be a limiting factor in lattice design. The phase advances become larger than those of uniform dipoles. Although they seem not to be prohibitively large, the larger phase advances make the lattice harder to realize. Even though it may be hard to achieve the theoretical minimum emittance of a nonuniform dipole (true for uniform dipoles as well), nonuniform dipoles are worthwhile to explore for reducing emittance, given the significant reduction in the theoretical minimum emittance.

For light-source applications, nonuniform dipoles seem to benefit multibend lattices most because the inner dipoles can take advantage of the larger emittance reduction from a symmetric TME dipole. The shorter but stronger constant-field segment in an optimal nonuniform dipole can generate harder $\mathrm{x}$ rays and provide more localized sources. Such stronger "superbends" have been used in low- and medium-energy light sources such as ALS [15] and SLS, although not for emittance reduction. Thus, it is natural to optimize such superbends for lower emittance.

In addition to storage rings, there are other accelerators that can take advantage of such minimum-emittance arcs to better preserve beam emittance during beam transport through a long arc or even a few turns of a ring without constraints for storing the beam. For example, future light sources based on either energy-recovery linac or recirculating linac can potentially benefit from reduced emittance growth in the arcs with nonuniform bends.

\section{ACKNOWLEDGMENTS}

The authors thank M. Borland and Q. Qin for introducing Y. Wang and Y. Peng, respectively, to work on this subject. Thanks to L. Emery for support and helpful discussions. C.-x. Wang would also like to acknowledge informative discussion with Y. Cai of SLAC at IPAC10. This work was supported by U.S. Department of Energy, Office of Science, Office of Basic Energy Sciences, under Contract No. DE-AC02-06CH11357.

\section{APPENDIX A: ANALYTICALLY SOLVED BENDING PROFILES}

Ignoring the weak focusing effect in a dipole, the transverse transport reduces to a drift whose matrix elements from the entrance $s_{0}$ to location $s$ are given by $M_{11}=1$ and $M_{12}=s-s_{0}$. Thus, the projected dispersion vector can be computed by the integrals $\hat{\boldsymbol{\xi}}=\left\{-\int_{s_{0}}^{s} h(s) \times\right.$ $\left.\left(s-s_{0}\right) d s, \int_{s_{0}}^{s} h(s) d s\right\}^{T}$. Inserting this into Eq. (10) leads to $|A|$ and $c$. Closed-form expressions can be obtained for simple profiles such as linearly or exponentially increasing bending radius [3].

\section{Linearly increasing profile}

For a linearly increasing bending radius, the normalized (to bending angle $L / \rho_{0}$ ) bending-radius profile reads 
TABLE I. Potential performance of linearly increasing bending-radius profile in Eq. (A1) at several peak-field values. All quantities are normalized by equivalent uniform dipoles of equal length and bending angle, indicated by the caret on $\mathcal{F}$.

\begin{tabular}{lrccccc}
\hline \hline$B_{\max } / B_{0}$ & \multicolumn{1}{c}{$\lambda$} & $\hat{\mathcal{F}}_{A}$ & $\hat{\mathcal{F}}_{c}$ & $\hat{\mathcal{F}}_{A} \hat{\mathcal{F}}_{c}$ & $1 / \hat{\mathcal{F}}_{\mathrm{TME}}$ & $1 / \hat{\mathcal{F}}_{\mathrm{EME}}$ \\
\hline 2.00 & 2.51 & 0.61 & 1.45 & 0.88 & 1.13 & 1.38 \\
3.30 & 6.76 & 0.45 & 1.65 & 0.74 & 1.35 & 1.75 \\
4.00 & 9.35 & 0.40 & 1.71 & 0.69 & 1.46 & 1.92 \\
6.00 & 17.51 & 0.32 & 1.80 & 0.58 & 1.73 & 2.32 \\
8.00 & 26.52 & 0.28 & 1.85 & 0.51 & 1.95 & 2.64 \\
\hline \hline
\end{tabular}

$$
\rho(s)=\rho_{0} \frac{\log (1+\lambda)}{\lambda}\left(1+\lambda \frac{s}{L}\right)
$$

with $0 \leq s \leq L$. The peak-field ratio is $\rho_{0} / \rho_{\min }=$ $\rho_{0} / \rho(0)=\lambda / \log (1+\lambda)$. This profile yields the projected dispersion vector as

$$
\hat{\boldsymbol{\xi}}(s)=\left[\begin{array}{c}
-\frac{L^{2}}{\rho_{0}} \frac{\lambda(s / L)-\log [1+\lambda(s / L)]}{\lambda \log (1+\lambda)} \\
\frac{L}{\rho_{0}} \frac{\log [1+\lambda(s / L)]}{\log (1+\lambda)}
\end{array}\right] .
$$

Inserting this into Eq. (10) and working out the integrals, we obtain the closed-form expressions

$$
\begin{gathered}
\hat{\mathcal{F}}_{A} \equiv \frac{2 \sqrt{|A|}}{\theta^{3} / 4 \sqrt{15}}=\frac{2 \sqrt{15 f_{\lambda}}}{\lambda(1+\lambda) \log (1+\lambda)^{3}}, \\
\hat{\mathcal{F}}_{c} \equiv \frac{\sqrt{1-c}}{1 / 3}=3 \sqrt{\frac{2(1+\lambda)^{2} g_{\lambda}}{\lambda(2+\lambda) f_{\lambda}}}
\end{gathered}
$$

where $f_{\lambda}=4(1+\lambda) \log (1+\lambda)\{\lambda[6+\lambda(9+\lambda)]-(1+\lambda) \times$ $\log (1+\lambda)[3+2 \log (1+\lambda)]\}-\lambda^{2}(2+3 \lambda)(6+5 \lambda)$ and $g_{\lambda}=$ $\lambda^{2}\left(12+12 \lambda+\lambda^{2}\right) \log (1+\lambda)-4 \lambda^{3}(2+\lambda)-4(1+\lambda)^{2} \times$ $\log (1+\lambda)^{3}$. Using these expressions, a few examples are computed and shown in Table I for comparison. The results for $B_{\max } / B_{0}=3.3$ agree with Table 1 of [3]. Comparison of the other results with the optimal solutions in Figs. 1 and 2 shows inferior performance of this oversimplified profile.

\section{Symmetric linearly increasing profile}

Similarly, for a bending radius that increases linearly and symmetrically from the dipole center, the normalized profile is given by

$$
\rho(s)=\rho_{0} \frac{\log (1+\lambda)}{\lambda}\left(1+2 \lambda \frac{|s|}{L}\right)
$$

with $-L / 2 \leq s \leq L / 2$. The projected dispersion vector works out as

$$
\hat{\boldsymbol{\xi}}(s)=\left[\begin{array}{c}
-\frac{L^{2}}{4 \rho_{0}} \frac{\lambda(1+2|s| / L)+\log [(1+2 \lambda|s| / L) /(1+\lambda)]}{\lambda \log (1+\lambda)} \\
\frac{L}{2 \rho_{0}}\left[1+\operatorname{sgn}(s) \frac{\log (1+2 \lambda|s| / L)}{\log (1+\lambda)}\right]
\end{array}\right] .
$$

TABLE II. Potential performance of symmetric linearly increasing bending-radius profile in Eq. (A5) at several peak-field values. All quantities are normalized by equivalent uniform dipoles of equal length and bending angle, indicated by the caret on $\mathcal{F}$.

\begin{tabular}{lcccccc}
\hline \hline$B_{\max } / B_{0}$ & \multicolumn{1}{c}{$\lambda$} & $\hat{\mathcal{F}}_{A}$ & $\hat{\mathcal{F}}_{c}$ & $\hat{\mathcal{F}}_{A} \hat{\mathcal{F}}_{c}$ & $1 / \hat{\mathcal{F}}_{\mathrm{TME}}$ & $1 / \hat{\mathcal{F}}_{\mathrm{EME}}$ \\
\hline 2.0 & 2.51 & 0.81 & 0.59 & 0.48 & 2.10 & 1.45 \\
3.3 & 6.76 & 0.78 & 0.38 & 0.30 & 3.36 & 1.63 \\
4.0 & 9.35 & 0.79 & 0.32 & 0.25 & 3.97 & 1.65 \\
6.0 & 17.51 & 0.84 & 0.22 & 0.18 & 5.47 & 1.61 \\
8.0 & 26.52 & 0.90 & 0.16 & 0.15 & 6.72 & 1.53 \\
\hline \hline
\end{tabular}

Calculating the integrals in Eq. (10), we obtain the closedform expressions

$$
\hat{\mathcal{F}}_{A}=\frac{\sqrt{15 f_{\lambda}}}{4 \lambda(1+\lambda) \log (1+\lambda)^{3}}
$$

$$
\hat{\mathcal{F}}_{c}=3 \sqrt{\frac{\left[\lambda^{2}+2 \lambda-2 \log (1+\lambda)-2 \log (1+\lambda)^{2}\right] g_{\lambda}}{2 \lambda(2+\lambda) f_{\lambda}}},
$$

where $\quad f_{\lambda}=\lambda^{2}(2+\lambda)^{2}\left(2 \lambda^{2}-2 \lambda-3\right)+\log (1+\lambda) \times$ $\left\{2 \lambda\left(12+18 \lambda-7 \lambda^{3}-2 \lambda^{4}\right)+\log (1+\lambda)\left[-12-5 \lambda^{3}(8+\right.\right.$ $\left.\left.5 \lambda)+8(1+\lambda)^{2}\left[-2+5 \lambda+\lambda^{2}-\log (1+\lambda)\right] \log (1+\lambda)\right]\right\}$ and $\quad g_{\lambda}=4(1+\lambda)^{2}\left[2 \lambda^{2}+8 \lambda-\log (1+\lambda)\right] \log (1+\lambda)-$ $\lambda^{2}\left(23 \lambda^{2}+52 \lambda+28\right)$. For comparison, a few examples are shown in Table II. Again, the results for $B_{\max } / B_{0}=3.3$ agree with Table 1 of [3], and the performance of this profile is inferior to the optimal solutions in Fig. 1.

[1] J. Guo and T. Raubenheimer, in Proceedings of the 8th European Particle Accelerator Conference, Paris, 2002 (EPS-IGA and CERN, Geneva, 2002), p. 1136.

[2] Y. Papaphilippou and P. Elleaume, in Proceedings of the 21st Particle Accelerator Conference, Knoxville, 2005 (IEEE, Piscataway, NJ, 2005), p. 2086.

[3] R. Nagaoka and A. F. Wrulich, Nucl. Instrum. Methods Phys. Res., Sect. A 575, 292 (2007).

[4] A. Streun, PSI Internal Report No. SLS-TME-TA-20060297, 2007.

[5] C.-x. Wang, Phys. Rev. ST Accel. Beams 12, 061001 (2009).

[6] H. Tanaka and A. Ando, Nucl. Instrum. Methods Phys. Res., Sect. A 369, 312 (1996).

[7] See, for example, http://en.wikipedia.org/wiki/ Genetic_algorithm.

[8] See, for example, S. Russenschuck, in Computational Electromagnetics International Journal of Applied Electromagnetics in Materials 4 (Elsevier, New York, 1993); Divisional Report No. AT/92-27, CERN LHCNote 205, Geneva; S. Ramberger, S. Russenschuck: Genetic Algorithms with Niching for Conceptional Design Studies, COMPUMAG, Rio, 1997. 
[9] I. V. Bazarov and C.K. Sinclair, Phys. Rev. ST Accel. Beams 8, 034202 (2005).

[10] K. Deb, A. Pratap, S. Agarwal, and T. Meyarivan, IEEE TEC 6, 182 (2002).

[11] R. Gruna, "Evolutionary Multiobjective Optimization" from The Wolfram Demonstration Project, http://demonstrations .wolfram.com/EvolutionaryMultiobjectiveOptimization.
[12] D. Levine, Report No. ANL-95/18, 1996.

[13] P. Emma and T. Raubenhemier, Phys. Rev. ST Accel. Beams 4, 021001 (2001).

[14] S. Y. Lee, Phys. Rev. E 54, 1940 (1996).

[15] D. Robin et al., in Proceedings of the 19th Particle Accelerator Conference, Chicago, Illinois, 2001 (IEEE, Piscataway, NJ, 2001), p. 2632. 